\title{
Ultrahigh Vacuum Optical Spectroscopy of Chemically Functionalized Graphene Nanoribbons
}

A Grüneis, BV Senkovskiy, AV Fedorov, M Hell, and S Michel, Universität zu Köln, Köln, Germany

(C) 2017 Elsevier Inc. All rights reserved.

\begin{tabular}{lr}
\hline Introduction & 1 \\
Experimental Setup & 1 \\
Graphene Nanoribbons & 2 \\
Ultrahigh Vacuum Optical Spectroscopy & 3 \\
Ultrahigh-Vacuum Raman Spectroscopy & 3 \\
Ultrahigh-Vacuum Photoluminescence & 6 \\
Conclusions and Outlook & 6 \\
Acknowledgments & 7 \\
References & 7
\end{tabular}

\section{Introduction}

Optical spectroscopy is a bulk sensitive technique and probes samples up to depths of several hundred nanometers. As such, the technique is rarely carried out in an ultrahigh vacuum (UHV) environment. However, when the dimensions of the sample are reduced to the atomic scale, for example, in layered materials, the surface plays an extremely important role. In this case, the experiment has to be performed with an intact surface, which can be achieved in UHV conditions. Here we present a simple experimental setup that is based on a commercially available Raman spectrometer which is interfaced to a home-built optical chamber. We apply optical spectroscopy (Raman and photoluminescence) in UHV and show that it can provide crucial information on the physical properties of surfaces. UHV optical spectroscopy can be carried out as a stand-alone method or in combination with established surface science methods such as low-energy electron diffraction (LEED), scanning tunneling microscopy (STM), and photoemission. Raman spectroscopy in the UHV has a long standing history in the context of surface-enhanced Raman scattering (SERS) ${ }^{1}$ and tip-enhanced Raman scattering. ${ }^{2}$ In SERS experiments of molecules, a molecule film is evaporated onto an in situ prepared film of Ag islands. The Ag islands are responsible to the Raman enhancement and in order to avoid Ag oxidation and to keep the sample clean, the measurements need to be carried out in UHV. Similarly, TERS measurements employing the tip of a UHV scanning tunneling microscope require the light to be coupled into a vacuum chamber and directed onto the sample.

We have chosen to carry out UHV Raman and photoluminescence spectroscopy on graphene nanoribbons (GNRs), ${ }^{3,4}$ i.e., about one nanometer wide stripes of graphene with a length of at least several tens of nanometers. Owing to their one-dimensionality and the atomic precision in synthesis, GNRs are currently a promising research direction. ${ }^{5}$ Their optical response is dominated by singularities in the joint density of states which cause a strong Raman response if the exciting light energy is equal to the singularity in the joint density of states. In fact, the response of even submonolayer coverages is sufficiently large so that neither SERS nor TERS need to be employed to collect Raman spectra with a large S/N ratio $>10$ in the order of one minute. One motivation for the application of UHV optical spectroscopy comes from the GNR synthesis which is performed by surface polymerization of precursor molecules on a noble metal single crystal substrate inside UHV. Here UHV optical spectroscopy can be a convenient means to monitor the polymerization process and to assess the quality of the synthesis product without exposing the substrate to ambient conditions. GNRs can readily be chemically functionalized using ionic and covalent dopants, and their physical properties are strongly affected by the type of functionalization. Probing the modification of optical properties upon doping in a controlled manner (e.g., by stepwise evaporation of a dopant) clearly requires an in situ UHV setup. The present paper addresses these aspects and is organized as follows. Section "Experimental Setup" briefly introduces the experimental setup and Section "Graphene Nanoribbons" explains the physical properties of $N=7$ armchair graphene nanoribbons. Section "UltraHigh Vacuum Optical Spectroscopy" shows results on UHV-Raman and photoluminescence spectroscopies of GNRs. Finally in Section "Conclusions and Outlook", we provide an outlook into further developments of this technique and application to other samples.

\section{Experimental Setup}

The experimental setup consists of a loadlock and preparation chamber with standard equipment (LEED, sputter gun, sample heater, and Knudsen cells for evaporation of molecules) and an analysis chamber into which light can be coupled in and out via an inverted flange. The analysis chamber and the laser path are shown in Fig. 1 together with a photograph of the setup. On the air-side of the inverted flange a long-working distance microscope objective is mounted. The working distance between the vacuum side of the window of the inverted flansch and the sample can be as small as a few millimeters. Any standard Raman or 

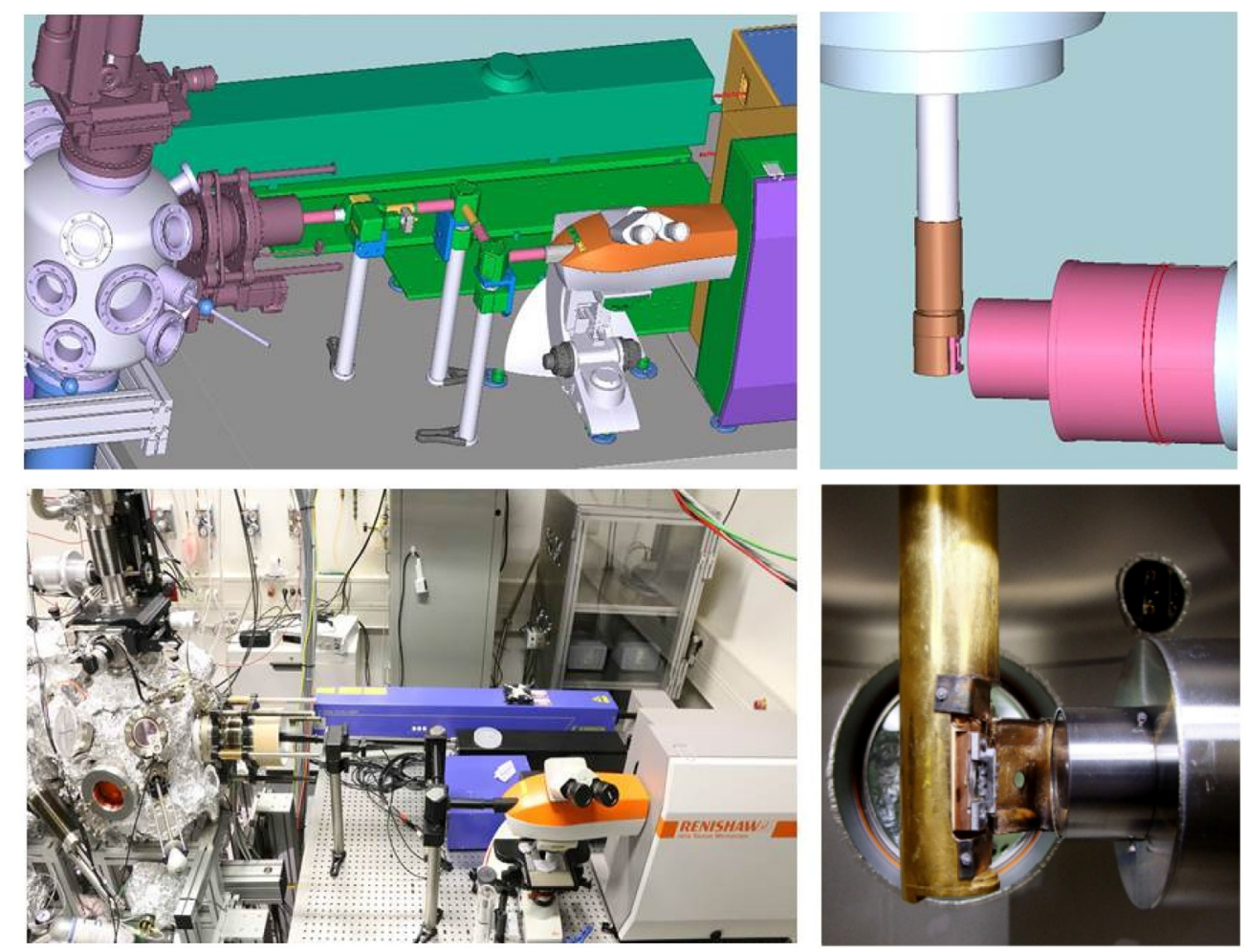

Fig. 1 Upper panel: Technical drawing of the UHV Raman setup and view of the optical flange in front of the cryogenic manipulator. Lower panel: photographs of the setup.

photoluminescence spectrometer can be used to analyze the scattered light. After coupling out the beam from the spectrometer (Renishaw) we use two mirrors to direct the beam into the analysis chamber. The beam path of the scattered light is identical to the incoming light. Laser wavelengths of $\lambda=633,532,442$, and $325 \mathrm{~nm}$ are available from three lasers. The samples can be cooled down to temperatures of about $4 \mathrm{~K}$ using a lHe flow cryostat. The pressure in the analysis chamber is less than $1 \times 10^{-10} \mathrm{mbar}$ which ensures long lifetimes of sensitive samples. Typically, a GNR sample is prepared in the preparation chamber by a surface polymerization procedure ${ }^{4}$ and then transferred into the analysis chamber. Here, the alkali metal doping is carried out by stepwise evaporation of alkali atoms onto the sample surface. Similarly the hydrogenation is carried out by cracking $\mathrm{H}_{2}$ in a tungsten capillary. Hydrogen $\mathrm{sp}^{3}$ defects in graphene-based carbon materials can be readily induced by exposure to a beam of atomic hydrogen for a defined time duration which form $\mathrm{C}-\mathrm{H}$ bonds in the basal plane of graphene and perpendicular to the nanotube walls. ${ }^{6-10}$ The spatial resolution is determined by the size of the laser spot and the vibrations of the system and is equal to $\sim 4 \mu \mathrm{m}$ for the setup described. As the sample manipulator is fully motorized in the plane parallel to the optical window, automated Raman mapping can be performed inside a UHV chamber. This is useful, for example, for determining the sample homogeneity.

\section{Graphene Nanoribbons}

One-dimensional graphene nanoribbons combine the best attributes of the nanotube and graphene worlds. They posses all the 1D properties plus they exist as highly uniform monochiral samples thanks to UHV bottom-up nanofabrication techniques. ${ }^{3,4,11-13}$ Fig. 2 depicts a sketch of the synthesis steps which involve molecule deposition on a Au surface, a first annealing to form polymer

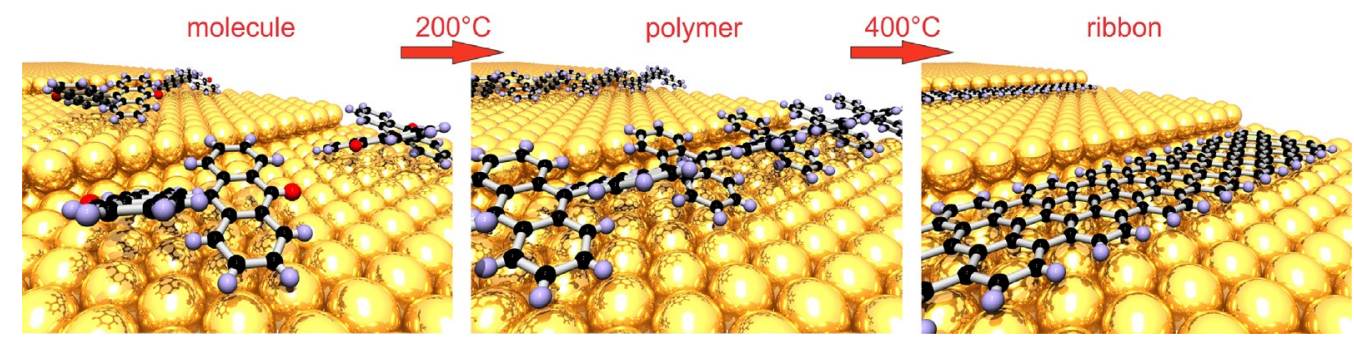

Fig. 2 Sketch of the synthesis steps needed for aligned $N=7$ armchair graphene nanoribbon growth on $\mathrm{Au}(788)$. Left: precursor molecules deposited on the surface. Middle: First annealing to about $200^{\circ} \mathrm{C}$ to form polymer chains. Right: Second annealing to about $400^{\circ} \mathrm{C}$ to form nanoribbons. 


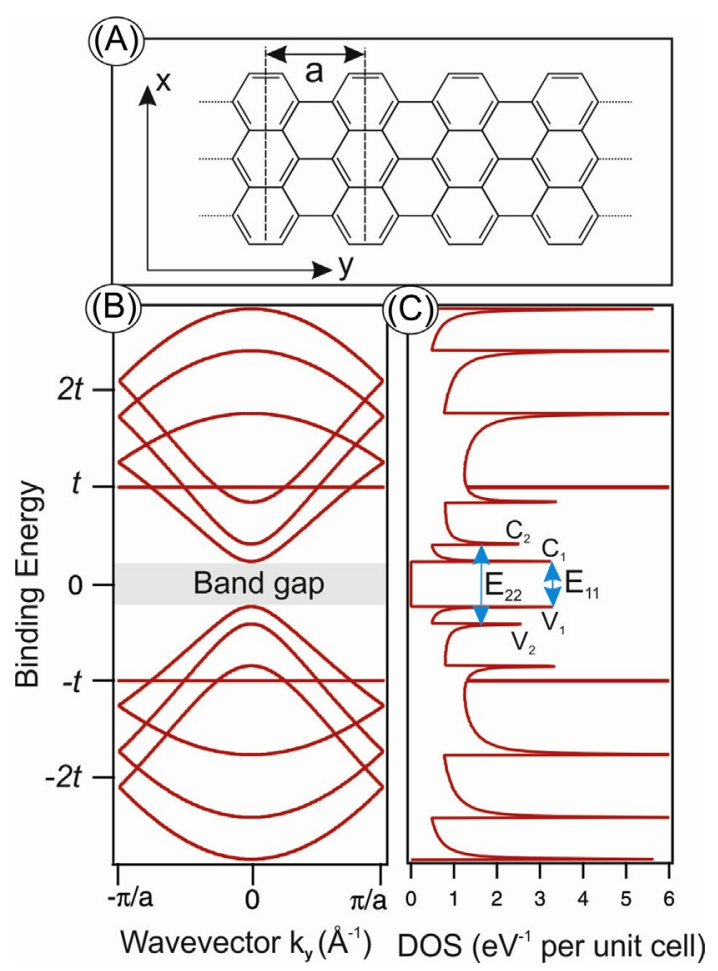

Fig. 3 (A) Structure and unit cell of a 7-AGNR (not showing the hydrogen atoms along the ribbon edge). The lattice constant is a. (B) electronic energy band dispersion and (C) density of states (DOS) of 7-AGNRs form the first nearest-neighbor tight-binding model, where $t$ is the transfer integral and $k_{y}$ is the wavevector along the ribbon length.

chains and second annealing to perform dehydrogenation. The ability to tune the band gap ${ }^{11,14}$ in semiconducting GNRs by varying the ribbon width via the choice of the precursor molecule and the ease of producing high-quality ohmic contacts makes them perfectly suited for production of nanoscale devices. ${ }^{15,16}$ The resonance Raman and photoluminescence measurements shown later in this manuscript can be rationalized qualitatively by tight-binding calculations of the electronic structure. In Fig. $3 \mathrm{~A}$ we depict the unit cell of a 7-AGNR with 14 carbon atoms that we considered for the simple nearest-neighbor tight-binding calculation with one free parameter (the transfer integral). ${ }^{17}$ Here we do not show the hydrogen atoms along the ribbon edge. The results for the electron energy dispersion relations and the density of electronic states are shown in Fig. 3B and C. Due to the 1D nature of GNRs their electronic density of states (DOS) is dominated by a series of van Hove singularities (VHSs). In accord with carbon nanotube literature ${ }^{17}$ we label the energies of the first and the second optical transitions between equal subbands as $\mathrm{E}_{11}$ and $E_{22}$. Furthermore we label the first and the second valence band as $V_{1}$ and $V_{2}$ and the first and the second conduction band $C_{1}$ and $\mathrm{C}_{2}$. Using a matching laser energy one can excite resonantly optical transitions between two VHSs. The $\mathrm{E}_{22}$ optical transition energy for 7-AGNRs/Au(788) and $\mathrm{Au}(111)$ was experimentally found to be equal to $2.3 \mathrm{eV}$. ${ }^{18}$ We will take advantage of the large optical response at the van Hove singularity in the following to perform resonance Raman spectroscopy with a $2.33 \mathrm{eV}$ laser excitation exciting 7-AGNRs at the $\mathrm{E}_{22}$ transition.

\section{Ultrahigh Vacuum Optical Spectroscopy \\ Ultrahigh-Vacuum Raman Spectroscopy}

We first discuss the Raman spectrum of pristine 7-AGNRs in terms of the one-dimensional phonon dispersion relations. For assignment of the experimentally observed Raman peaks of 7-AGNRs to phonon eigenvectors we perform calculations within force constant model. ${ }^{17}$ Fig. 4A depicts the calculated phonon dispersion relation for a set of force constants and bond polarizability values that have been used for graphene. ${ }^{17}$ The obtained results are consistent with previously published data. ${ }^{19}$ Open circles in Fig. 4A indicate the six Raman active phonons as theoretically predicted by bond-polarizability theory. Clearly, the bond polarizability theory is not taking into account the resonant nature of the process and as such fails to predict quantitative Raman intensities and second-order Raman processes. However, its predictions regarding the first-order Raman modes work very well for graphene nanoribbons. ${ }^{20}$ Fig. $4 \mathrm{~B}$ depicts the Raman spectra together with theoretically predicted Raman active phonons. The good agreement of experimental data with theoretical calculations allows for an assignment of the phonon eigenvectors. The fingerprint of 7-AGNRs is the so-called radial breathing like mode (RBLM) at $397 \mathrm{~cm}^{-1}$, and this mode is related to the ribbon width expansion. ${ }^{19,21,22}$ Another breathing-like mode is found at $957 \mathrm{~cm}^{-1}$ and was also predicted for 19 -AGNRs. ${ }^{20}$ 

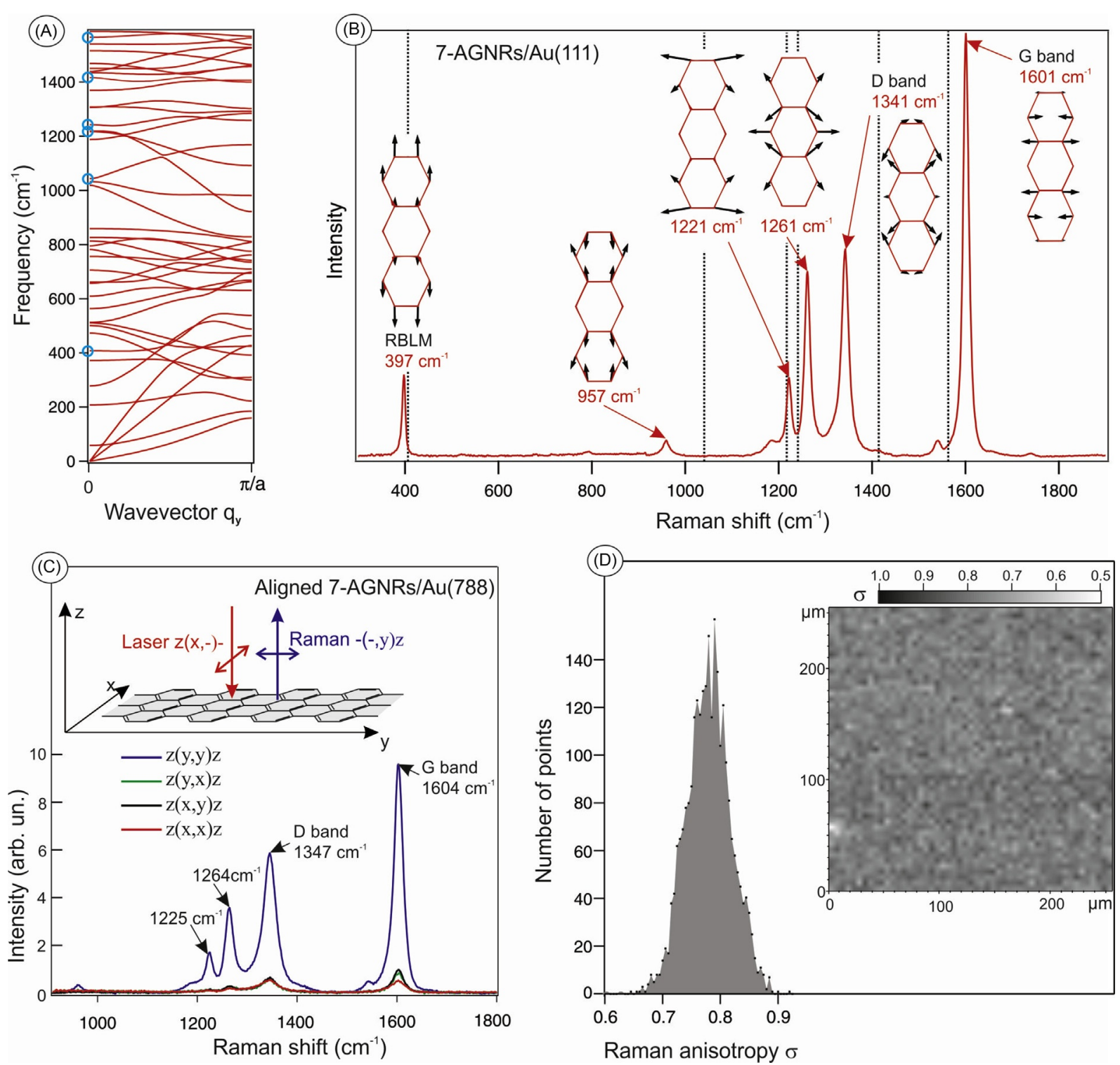

Fig. 4 (A) Phonon dispersion relation for 7-AGNRs obtained in a force constant model using four nearest-neighbor atoms. The Raman active modes at $q_{y}=0$ are shown by the blue open circles. (B) Raman spectra of pristine 7-AGNRs on $\mathrm{Au}(111)$ measured with a $532 \mathrm{~nm}$ laser. Atomic displacements of the atoms in the unit cell and calculated positions of the Raman active modes (vertical lines) are shown. (C) Polarized Raman spectra of aligned 7-AGNRs on $\mathrm{Au}(788)$ measured in $180^{\circ}$ back scattering geometry. (D) Histogram plot of the Raman anisotropy $(\sigma)$ extracted over the $250 \times 250 \mu \mathrm{m}$ area, where $\sigma$ was measured with $0.5 \mu \mathrm{m}$ step. Copyright information: Panels (A) and (B) are taken from Senkovskiy, B. V. et al. Adv. Electron. Mater. 2017, 1600490.

Furthermore we observe features in the Raman spectra at 1221 and $1261 \mathrm{~cm}^{-1}$ which can be assigned to the theoretically predicted Raman phonons in a straightforward manner. The peak observed at $1341 \mathrm{~cm}^{-1}$ resembles many features of the $\mathrm{D}^{-b a n d^{23,24}}$ in sp ${ }^{2}$ carbon materials, i.e., its frequency is close to the D-band frequency of graphene and its intensity depends on the defect concentration. It has been put forward that in the case of AGNRs due to the zone folding the D-band can exist both as a firstand second-order process. ${ }^{25}$ Finally we assign the longitudinal optical (LO) phonon to the G band at $1601 \mathrm{~cm}^{-1}$. In the present case, the transverse optical (TO) phonon is not visible in the resonance Raman spectrum. ${ }^{26}$ However, it is possible to observe it under different conditions with respect to laser energy and ribbon geometry. Interestingly, despite the resonant nature of the experiment the theoretical model is capable of reproducing all experimentally observed Raman modes similar to the bondpolarizability calculations for carbon nanotubes. ${ }^{17}$ The differences in frequencies may occur due to the set of force constants that have been fitted to graphite. ${ }^{17}$ Graphene nanoribbons might have different values of the force constants due to their narrow width, varying bond-lengths and substrate effects.

The good alignment of the nanoribbons allows to probe their optical anisotropy in polarized Raman measurements. The scattering geometry is shown in Fig. 4C. Here we use the Porto notation, e.g., $z(y, y) z$ indicates that the incident and scattered light propagate in $z$ direction and the incident and scattered light are polarized along the $y$ direction, i.e., along the direction parallel to 
the terraces $(\mathrm{Au}[011])$. Similarly $z(x, x) z$ indicates a polarization of incident and scattered light perpendicular to the terraces. It is clear that the GNRs are aligned almost perfectly parallel to the terraces. It can be seen that the Raman intensity ratio for $z(y, y) z$ and $z(x, x) z$ is equal to $\sim 10$. This anisotropy is governed by the dipole selection rules ${ }^{27}$ which state that for armchair nanoribbons the $\mathrm{E}_{i i}$ optical transition is allowed only for the light polarized along the ribbon axis. Another factor that affects the intensity is the depolarization effect which strongly suppresses the absorption of the perpendicularly polarized light. ${ }^{28}$

In order to probe the spatial dependence of the optical anisotropy over large areas of $\sim 100 \mathrm{~s}$ of $\mu \mathrm{m}$, we have carried out polarized Raman mapping: two polarized Raman spectra with light polarization along and perpendicular the step edges of the single crystal are recorded every $500 \mathrm{~nm}$. We integrate the G band Raman intensities for the two polarizations and call them $I_{||}$and $I_{\perp}$, respectively. For each point we plot the Raman anisotropy $\sigma$ which we define as $\sigma=\left(I_{\|}-I_{\perp}\right) /\left(I_{||}+I_{\perp}\right)$. Fig. 4D shows a histogram plot of the values of $\sigma$ obtained over the whole macroscopic sample area shown in the right side of this figure. It can be seen that a vast majority of $80 \%$ of the GNRs have an optical anisotropy of the axis along the step edges in the range of $0.73-0.83$. Thus, the optical anisotropy, i.e., the nanoribbon alignment prevails over macroscopic areas. This highlights that aligned GNRs can be used in applications such as polarizers or optical absorbers. Fig. 5 displays a sketch of how different misorientations of the nanoribbon with respect to the terraces would affect $\sigma$.

To investigate the effect of electron doping by alkali metals on the vibrational spectrum of 7-AGNRs, we performed in situ UHV Raman measurements of Li-doped GNRs. ${ }^{29}$ In order to confirm the Fermi level shift we have also performed angle-resolved photoemission measurements ${ }^{29}$ (not shown here) which confirm that we have induced a semiconductor-to-metal transition. Let us now discuss how electron doping by alkali metals affect the intensities and positions of the Raman modes of graphene nanoribbons. The large intensity we observe in the case of undoped nanoribbons is a consequence of the resonant nature of the Raman process and the fact that the laser energy is equal to the $E_{22}$ transition. ${ }^{30}$ The electron doping by alkali atoms populates the conduction band and can therefore block optical transitions (Pauli blocking). The other effect of doping is that the dielectric environment and hence the exciton binding energy is changed. This changes the resonance condition and can therefore also affect the intensity. Regarding the position of the Raman mode, there are two effects which stem from the electron doping. One is that upon population of antibonding conduction band states, the lattice expands, and the force constants between atoms on different sites become smaller. This leads to a reduction of the Raman mode frequency. ${ }^{31}$ The second effect of doping on the Raman mode positions is more subtle and is due to nonadiabatic effects. ${ }^{32}$ These are corrections to the phonon frequency by the phonon selfenergy. ${ }^{33}$ For graphene nanoribbons, calculations of the phonon self-energy show that these corrections cause an upshift of the phonon frequency (see supporting info of Ref. 27). Thus, the effects of lattice expansion and nonadiabatic effects tend to cancel each other.

The UHV Raman spectra of 7-AGNRs on Au(788) before and after deposition of Li are shown in Fig. 6A. Li was evaporated onto the sample from commercial getter sources. After Li doping, a reduction in the Raman intensity by a factor of $\sim 20$ is observed which we attribute to Pauli blocking of the optical transition or the change of the resonance condition. We find that Li doping induces only very small changes in the Raman frequencies. We observe an energy upshift for the $\mathrm{G}$ peak by $2 \mathrm{~cm}^{-1}$, while the $D$ peak and the peak at $1260 \mathrm{~cm}^{-1}$ are down shifted by up to $8 \mathrm{~cm}^{-1}$.

In order to illustrate the importance of the laser wavelength, UHV Raman spectra of pristine GNRs are shown in Fig. 6B. It can be seen that the fingerprint mode of GNRs, the RBLM mode, cannot be clearly seen under this irradiation wavelength. Contrary, if the laser wavelength is in resonance, it is possible to even see overtones of G and D or the combination thereof (see Fig. 6C).
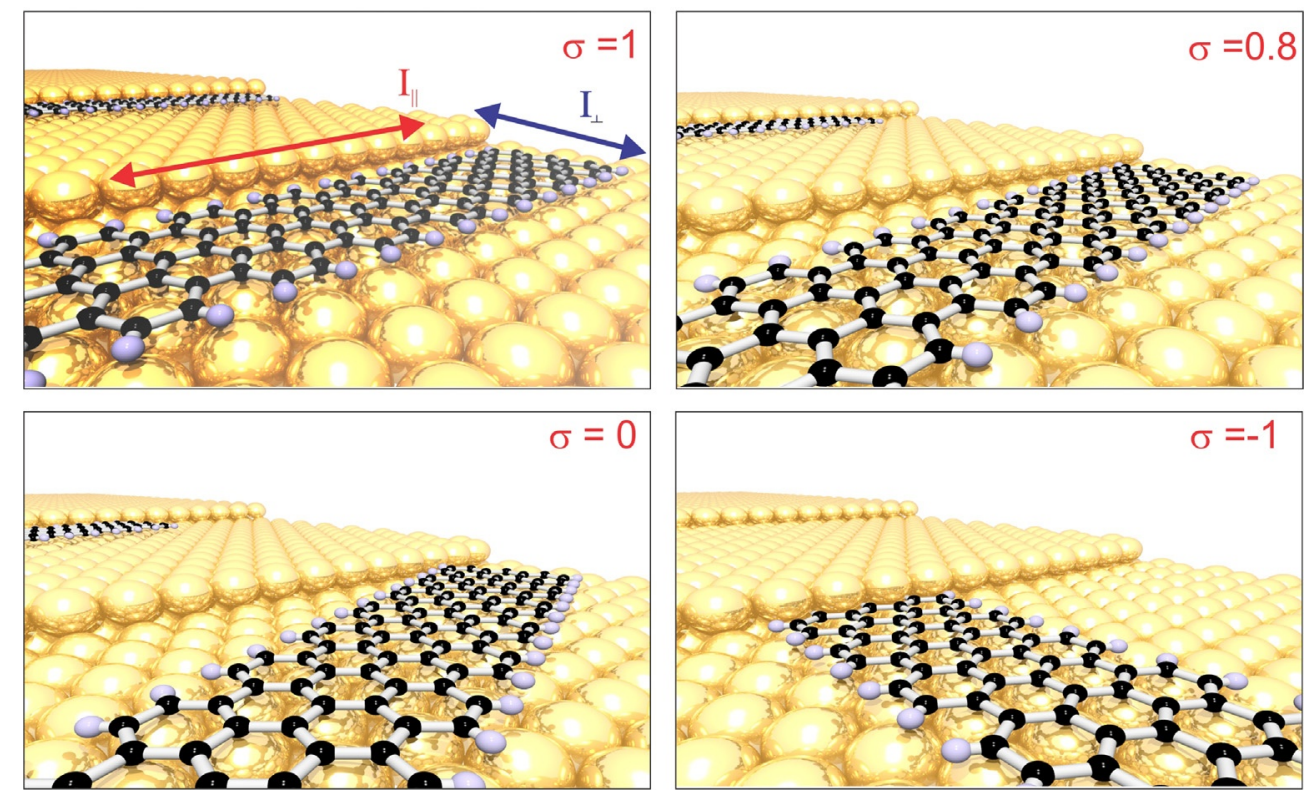

Fig. 5 Illustration of various nanoribbon orientations with respect to the step edges leading to different values of the Raman anisotropy $\sigma$. 

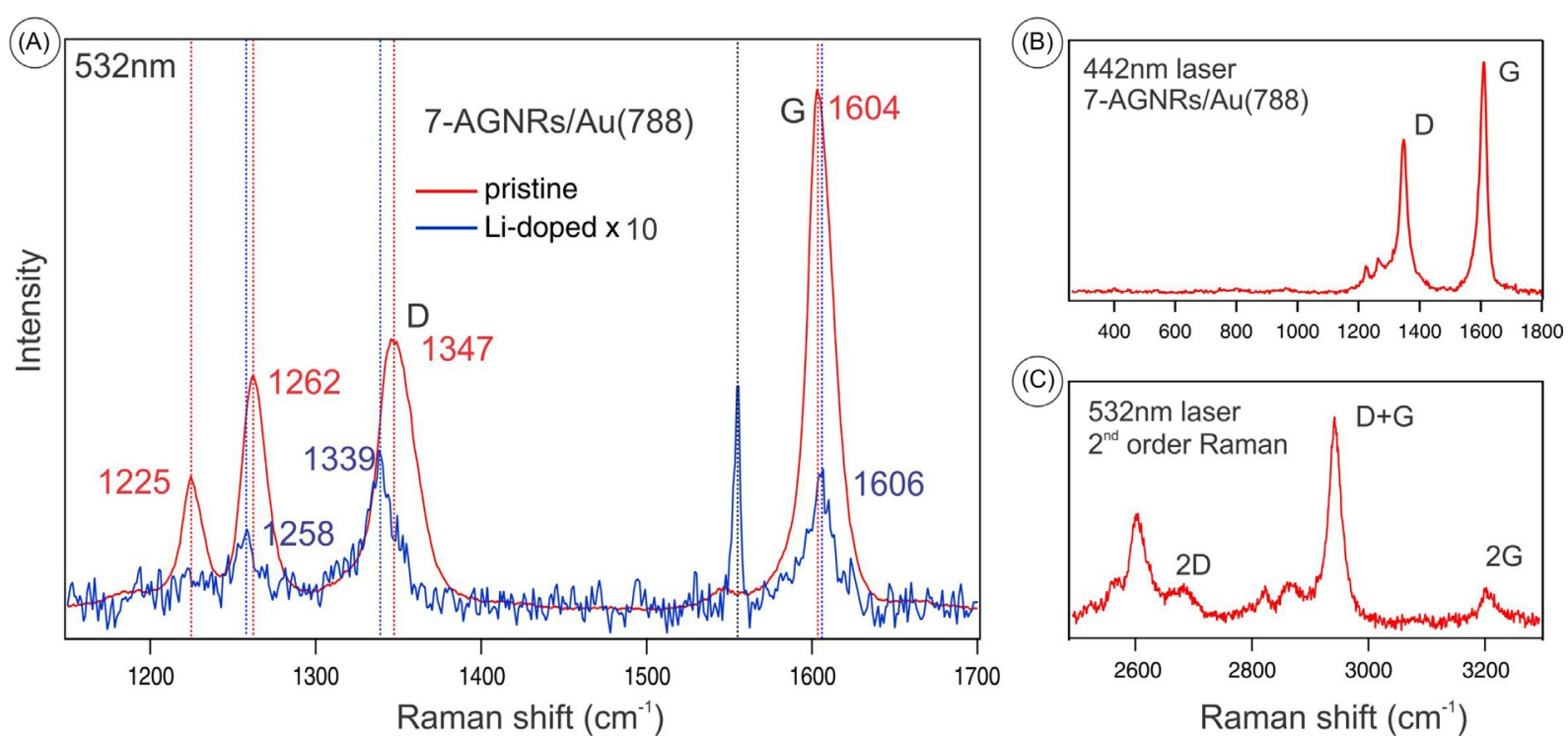

Fig. 6 (A) UHV Raman spectra of 7-AGNRs on Au(788) measured before and after Li deposition with a $532 \mathrm{~nm}$ laser. Taken from Senkovskiy, B. V. et al. Adv. Electron. Mater. 2017, 1600490. (B) UHV Raman spectrum of pristine 7-AGNRs using a $442 \mathrm{~nm}$ laser. (C) UHV Raman spectrum of the second order phonon modes in pristine GNRs using a $532 \mathrm{~nm}$ laser.

\section{Ultrahigh-Vacuum Photoluminescence}

In order to carry out photoluminescence, the GNR monolayer must be removed from the metal substrate as it would promote radiationless electron-hole recombination. To that end we applied the bubbling transfer method ${ }^{30}$ which was developed for CVD grown graphene. ${ }^{34,35}$ The advantage of this technique is, that it retains the GNR parallel alignment over hundreds of microns. Furthermore, it does not affect the single crystalline surface of the substrate so that it can be used for repeated transfers. ${ }^{36}$ The hydrogenation ${ }^{6-10}$ of transferred GNRs on $\mathrm{SiO}_{2} / \mathrm{Si}$ and the photoluminescence experiments were performed inside the UHV optical system without exposing the sample to air after hydrogenation. In such a well-defined experiment, the photoluminescence can be definitely ascribed to $\mathrm{sp}^{3}$ defects formed by $\mathrm{C}-\mathrm{H}$ bonds. Fig. 7A depicts the photoluminescence spectra of the sample inside UHV before hydrogenation measured with a $532 \mathrm{~nm}$ laser with light polarized parallel and perpendicular to the GNRs. The spectra exhibit strong Raman modes and their higher orders. It is seen that intrinsically photoluminescence from 7-AGNRs is very weak. Exposure to a beam of atomic hydrogen ${ }^{9,10}$ induces defects where a carbon atom forms three $\mathrm{C}-\mathrm{C}$ bonds and one $\mathrm{C}-\mathrm{H}$ bond, as illustrated in Fig. 7B. It can be seen from Fig. 7C that the hydrogenation ( $10 \mathrm{~min}$ exposure) leads to a strongly polarized photoluminescence peak with a maximum at around $1.8 \mathrm{eV}$. At the same time, the Raman signal from ribbons becomes weaker. We believe that this is due to the fact that the number of resonant $\mathrm{sp}^{2}$ bonds is reduced. We also observe disorder-induced broadening of the Raman lines. This is presumably due to the random nature of chemisorbed $\mathrm{H}$ atoms. Longer exposure (40 min) to atomic hydrogen corresponds to the maximum amount of hydrogen that can be chemisorbed to 7-AGNRs. It is important that the photoluminescence is polarized and therefore comes from the GNR. Since the energy of the photoluminesence is below the lowest optical transition and the PL/Raman signal ratio increases with hydrogen exposure. It is not a band gap luminescence but rather comes from defect levels that are energetically below the lowest excitonic state. ${ }^{30}$

\section{Conclusions and Outlook}

In summary we have applied Raman and photoluminescence spectroscopies in UHV to study the electronic and vibrational properties of air-sensitive, chemically functionalized GNRs. The signals are sufficiently strong so that monolayers of GNRs can be measured easily. We have shown that, upon alkali metal doping the Raman intensities decrease by a magnitude due to the loss of the resonance condition. The frequency of the Raman active phonon modes exhibits only small shifts which is explained by the opposite effects of lattice expansion and nonadiabatic effects on the Raman mode frequency. For the case of covalently functionalized GNRs by hydrogen defects, we found an increase in the photoluminescence after hydrogen exposure. Since the photoluminescence is polarized and has an energy below the optical band gap, it comes from defect levels which are located below the band gap. For future applications of UHV Raman and PL spectroscopy it would be interesting to apply it to alkali metal-doped ribbons of different width such as the $N=9$ graphene nanoribbons, ${ }^{37}$ alkali-doped graphene, or transition metal dichalcogenides such as $\mathrm{MoS}_{2}$. For the future development of the method, it would be interesting to perform also in situ electrical transport measurements along with Raman and photoluminescence. This would allow to probe the optical properties of doped materials in the superconducting or charge-density wave state. 

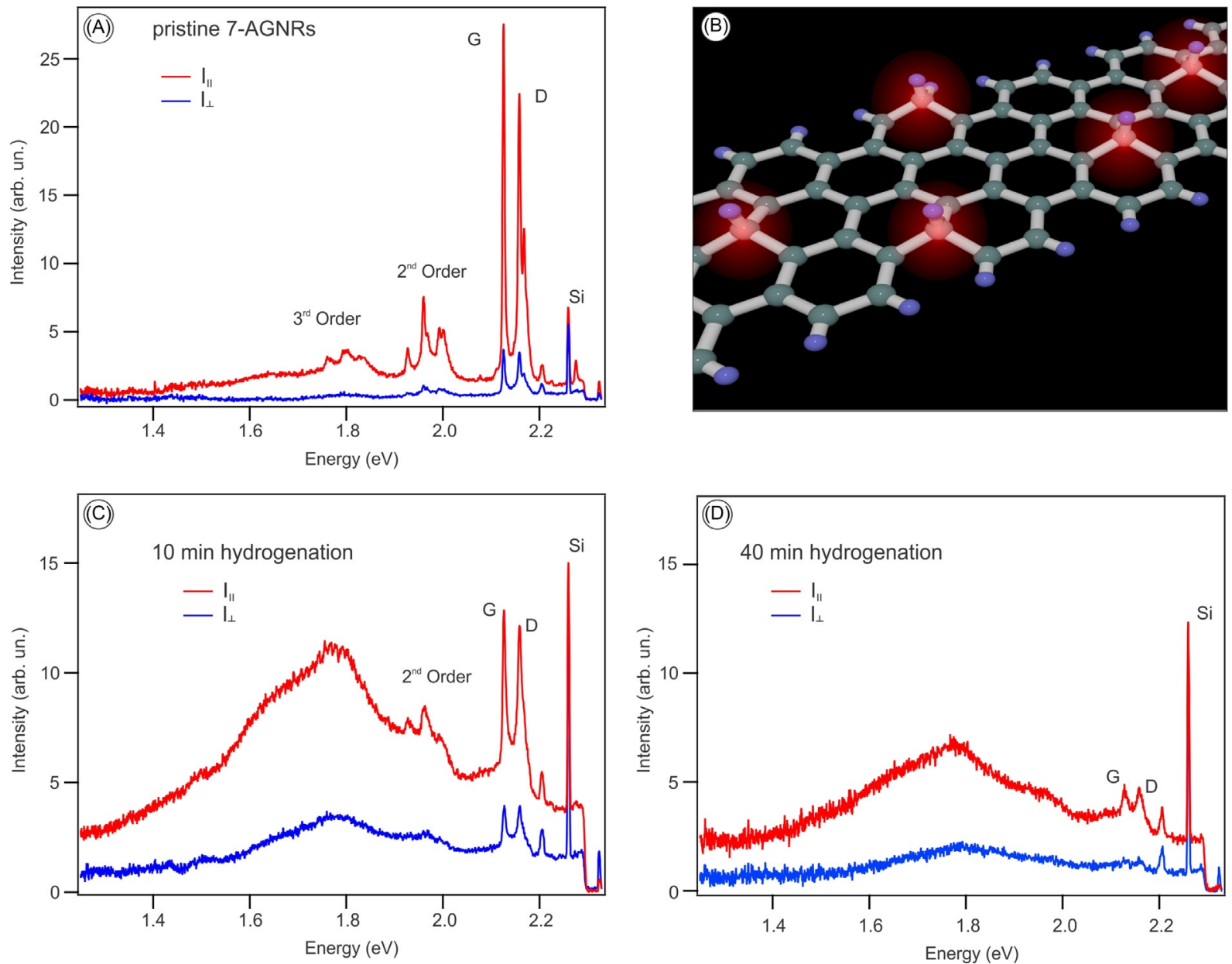

Fig. 7 (A) Photoluminescence and Raman spectra of transferred 7-AGNRs $/ \mathrm{SiO}_{2}$ measured inside a UHV chamber after annealing at $300^{\circ} \mathrm{C}$. Red and blue lines indicate spectrum recorded along $\left(I_{\|}\right)$and perpendicular $\left(I_{\perp}\right)$ to the alignment direction, respectively. (B) Sketch of the hydrogenated ribbons. (C, D) photoluminescence and Raman spectra of the system after 10 and 40 min of atomic hydrogen exposure. A polarized photoluminescence peak is induced by $\mathrm{sp}^{3}$ hydrogen defects. Taken from Senkovskiy, B. V. et al. Nano Lett. 2017, 17, 4029-4037.

\section{Acknowledgments}

A.G., B.V.S., A.V.F., M.H. and S.M. acknowledge the ERC grant no. 648589 "SUPER-2D," funding from DFG projects INST 216/808-1 FUGG and GR 3708/2-1.

\section{References}

1. Otto, A.; Mrozek, I.; Grabhorn, H.; Akemann, W. J. Phys. Condens. Matter 1992, 4, 1143.

2. Pozzi, E. A.; Goubert, G.; Chiang, N.; Jiang, N.; Chapman, C. T.; McAnally, M. 0.; Henry, A.I.; Seideman, T.; Schatz, G. C.; Hersam, M. C.; Duyne, R. P. V. Chem. Rev. 2017, 117, 4961-4982.

3. Grill, L.; Dyer, M.; Lafferentz, L.; Persson, M.; Peters, M. V.: Hecht, S. Nat. Nanotechnol. 2007, 2. 687-691.

4. Cai, J.; Ruffieux, P.; Jaafar, R.; Bieri, M.; Braun, T.; Blankenburg, S.; Muoth, M.; Seitsonen, A. P.; Saleh, M.; Feng, X.; Müllen, K.; Fasel, R. Nature 2010, 466, 470-473.

5. Talirz, L.; Ruffieux, P.; Fasel, R. Adv. Mater. 2016, 28, 6222-6231.

6. Nikitin, A.; Ogasawara, H.; Mann, D.; Denecke, R.; Zhang, Z.; Dai, H.; Cho, K.; Nilsson, A. Phys. Rev. Lett. 2005, 95, 225507.

7. Elias, D. C.; Nair, R. R.; Mohiuddin, T. M. G.; Morozov, S. V.; Blake, P.; Halsall, M. P.; Ferrari, A. C.; Boukhvalov, D. W.; Katsnelson, M. I.; Geim, A. K.; Novoselov, K. S. Science 2009, 323, 610-613.

8. Balog, R.; et al. Nat. Mater. 2010, 9, 315-319.

9. Haberer, D.; Vyalikh, D. V.: Taioli, S.; Dora, B.; Fariam, M.; Fink, J.; Marchenko, D.; Pichler, T.; Ziegler, K.; Simonucci, S.; Dresselhaus, M. S.; Knupfer, M.; Büchner, B. Grüneis, A. Nano Lett. 2010, 10, 3360-3366.

10. Haberer, D.; et al. Adv. Mater. 2011, 23, 4497.

11. Chen, Y.-C.; de Oteyza, D. G.; Pedramrazi, Z.; Chen, C.; Fischer, F. R.; Crommie, M. F. ACS Nano 2013, 7, 6123-6128.

12. Cloke, R. R.; Marangoni, T.; Nquyen, G. D.; Joshi, T.; Rizzo, D. J.; Bronner, C.; Cao, T.; Louie, S. G.; Crommie, M. F.; Fischer, F. R. J. Am. Chem. Soc. 2015, 137, 8872-8875

13. Kawai, S.; Saito, S.; Osumi, S.; Yamaguchi, S.; Foster, A. S.; Spijker, P.; Meyer, E. Nat. Commun. 2015, 6, 8098. 
14. Han, M. Y.; Ozyilmaz, B.; Zhang, Y.; Kim, P. Phys. Rev. Lett. 2007, 98, 206805.

15. Chen, Z.; Lin, Y.-M.; Rooks, M. J.; Avouris, P. Physica E: Low-dimensional Systems and Nanostructures. Phys. Rev. Lett. 2007, 40, 228-232. International Symposium on Nanometer-Scale Quantum Physics.

16. Li, X.; Wang, X.; Zhang, L.; Lee, S.; Dai, H. Science 2008, 319, 1229-1232.

17. Saito, R.; Dresselhaus, G.; Dresselhaus, M. S. Physical Properties of Carbon Nanotubes; Imperial College Press: London, 1998.

18. Denk, R.; Hohage, M.; Zeppenfeld, P.; Cai, J.; Pignedoli, C. A.; Söde, H.; Fasel, R.; Feng. X.; Müllen, K.; Wang. S.; Prezzi, D.; Ferretti, A.; Ruini, A.; Molinari, E.; Ruffieux, P. Nat. Commun. 2014, 5, 4253.

19. Sanders, G. D.; Nugraha, A. R. T.; Saito, R.; Stanton, C. J. Phys. Rev. B 2012, 85, 205401

20. Saito, R.; Furukawa, M.; Dresselhaus, G.; Dresselhaus, M. S. J. Phys. Condens. Matter 2010, 22, 334203.

21. Zhou, J.; Dong. In J. Applied Physics Letters; 91, 2007.

22. Vandescuren, M.; Hermet, P.; Meunier, V.; Henrard, L.; Lambin, P. Phys. Rev. B 2008, 78, 195401.

23. Thomsen, C.; Reich, S. Phys. Rev. Lett. 2000, 85, 5214-5217.

24. Ferrari, A. C.: Meyer, J. C.; Scardaci, V.: Casiraghi, C.: Lazzeri, M.; Mauri, F.; Piscanec, S.; Jiang, D.; Novoselov, K. S.: Roth, S.; Geim, A. K. Phys. Rev. Lett. 2006, $97,187401$.

25. Sasaki, K.; Kato, K.; Tokura, Y.; Suzuki, S.; Sogawa, T. Phys. Rev. B 2012, 85, 075437.

26. Sasaki, K.; Saito, R.; Wakabayashi, K.; Enoki, T. J. Phys. Soc. Jpn. 2010, 79, 044603.

27. Sasaki, K.; Kato, K.; Tokura, Y.; Oguri, K.; Sogawa, T. Phys. Rev. B 2011, 84, 085458.

28. Duesberg, G. S.; Loa, l.; Burghard, M.; Syassen, K.; Roth, S. Phys. Rev. Lett. 2000, 85, 5436-5439.

29. Senkovskiy, B. V.; et al. Adv. Electron. Mater. 2017, 1600490

30. Senkovskiy, B. V.; et al. Nano Lett. 2017, 17, 4029-4037.

31. Lazzeri, M.; Mauri, F. Phys. Rev. Lett. 2006, 97, 266407.

32. Pisana, S.; Lazzeri, M.; Casiraghi, C.; Novoselov, K.; Geim, A.; Ferrari, A.; Mauri, F. Nat. Mater. 2007, 6, 198

33. Caudal, N.; Saitta, A. M.; Lazzeri, M.; Mauri, F. Phys. Rev. B 2007, 75, 115423.

34. Gao, L.; Ren, W.; Xu, H.; Jin, L.; Wang, Z.; Ma, T.; Ma, L.-P.; Zhang, Z.; Fu, Q.; Peng, L.-M.; Bao, X.; Cheng, H.-M. Nat. Commun. 2012, 3, 699.

35. Wang, Y.; Zheng, Y.; Xu, X.; Dubuisson, E.; Bao, Q.; Lu, J.; Loh, K. P. ACS Nano 2011, 5, 9927-9933.

36. Rakic, I. S.; Capeta, D.; Plodinec, M.; Kralj, M. Carbon 2016, 96, 243-249.

37. Senkovskiy, B. V.; Haberer, D.; Usachov, D. Y.; Fedorov, A. V.; Ehlen, N.; Hell, M.; Petaccia, L.; Di Santo, G.; Durr, R. A.; Fischer, F. R.; Grüneis, A. Phys. Status Solidi (RRL) Rapid Res. Lett. 2017, 11, 1700157 